University of Wollongong

Research Online

Faculty of Engineering - Papers (Archive)

Faculty of Engineering and Information

Sciences

October 1996

\title{
Tunneling spectroscopy of hole plasmons in a valence-band quantum well
}

B. R. Neves

University of Nottingham, UK

T. J. Foster

University of Nottingham, UK

L. Eaves

University of Nottingham, UK

P. C. Main

University of Nottingham, UK

M. Henini

University of Nottingham, UK

See next page for additional authors

Follow this and additional works at: https://ro.uow.edu.au/engpapers

Part of the Engineering Commons

https://ro.uow.edu.au/engpapers/254

\section{Recommended Citation}

Neves, B. R.; Foster, T. J.; Eaves, L.; Main, P. C.; Henini, M.; Fisher, D. J.; Lerch, M. L.; Martin, A. D.; and Zhang, C.: Tunneling spectroscopy of hole plasmons in a valence-band quantum well 1996.

https://ro.uow.edu.au/engpapers/254

Research Online is the open access institutional repository for the University of Wollongong. For further information contact the UOW Library: research-pubs@uow.edu.au 


\section{Authors}

B. R. Neves, T. J. Foster, L. Eaves, P. C. Main, M. Henini, D. J. Fisher, M. L. Lerch, A. D. Martin, and C. Zhang 


\title{
Tunneling spectroscopy of hole plasmons in a valence-band quantum well
}

\author{
B. R. A. Neves, T. J. Foster, L. Eaves, P. C. Main, and M. Henini \\ Department of Physics, University of Nottingham, Nottingham, NG7 2RD, United Kingdom \\ D. J. Fisher, M. L. Lerch, A. D. Martin, and C. Zhang \\ Department of Physics, University of Wollongong, Wollongong NSW 2522, Australia \\ (Received 16 July 1996)
}

\begin{abstract}
We investigate the current-voltage characteristics of a $p$-doped resonant tunneling diode. In the voltage range slightly above the bias corresponding to resonant tunneling of holes into the first light-hole subband of the quantum well, we observe two satellite peaks which we attribute to plasmon-assisted tunneling transitions. A theoretical model is presented to account for these peaks. The model is based on the excitation of intrasubband and intersubband heavy-hole plasmons in the quantum well by hot holes injected close to the energy of the first light-hole subband. We also study the behavior of the satellites when a magnetic field is applied either parallel to or perpendicular to the current. [S0163-1829(96)51540-7]
\end{abstract}

Perhaps the most direct demonstration of the quantized nature of plasma excitations is the energy-loss spectrum of high-energy electrons injected into a metallic film where peaks are observed due to the excitation of bulk plasmons. ${ }^{1}$ Recently, an analogous effect has been observed due to plasmon excitation of the degenerate two-dimensional electron gas in the quantum well $(\mathrm{QW})$ of a double-barrier resonant tunneling diode (RTD). ${ }^{2}$ The RTD was constructed with tunnel barriers of different thickness so that a significant buildup of electron charge in the QW occurred when the device was biased for resonance. ${ }^{3,4}$ A satellite feature was observed in the current-voltage characteristics $I(V)$ which arose from the excitation of two-dimensional plasmons in the degenerate electron gas of the quantum well..$^{5}$ The satellite was observed at bias voltages slightly beyond the condition for resonant tunneling into the first electronic subband of the quantum well. The excitation energy of the plasmon is provided by the excess energy of electrons tunneling into the quantum well from the negatively biased emitter contact. In this bias range the current is not single valued due to an electrostatic effect associated with space-charge buildup in the quantum well at resonance. $^{2,6}$ This gives rise to a "voltage overhang" in $I(V)$ which leads to intrinsic current bistability when the RTD is measured with a conventional voltage sweep circuit. $^{3,4}$ To explore the region of $I(V)$ where the plasmon satellite occurs requires a circuit with negative output resistance (NOR). ${ }^{6}$

In this paper, we investigate the analogous process of hole tunneling in a $p$-type RTD, and observe two distinct satellite peaks which we attribute to the excitation of twodimensional (2D) hole plasmons in the QW. The special interest in studying this type of device is that the band structure of the hole states in the quantum well is more complex ${ }^{7,8}$ than that of electrons. We present a model to account for the current-voltage characteristics $I(V)$ and find significant contributions to the current from plasmon excitations due to both intrahole and interhole subband processes. We investigate the effect of magnetic field on the hole-plasmon features in $I(V)$.
A schematic band diagram of the device with an applied bias is shown in Fig. 1. It consists of a 4.5-nm emitter AlAs barrier and a 5.7-nm collector AlAs barrier enclosing a 4.2-nm GaAs quantum well. The asymmetric nature of the device gives rise to hole space-charge buildup in the well for resonances in one bias direction. Undoped GaAs spacer layers of $5.1 \mathrm{~nm}$ separate the barriers from graded $p$-type (Be doped) GaAs contact layers. Under bias, a two-dimensional hole gas (2DHG) accumulates on the positively biased (hole emitter) side of the device. The $I(V)$ characteristics reveal several resonant features corresponding to tunneling into heavy-hole $(\mathrm{HH} n)$ and light-hole $(\mathrm{LH} n)$ subbands of the quantum well. Here, $n$ is in the quantum number of the envelope function. Full details of the composition of the device are given in Ref. 7, which also describes a series of magne-

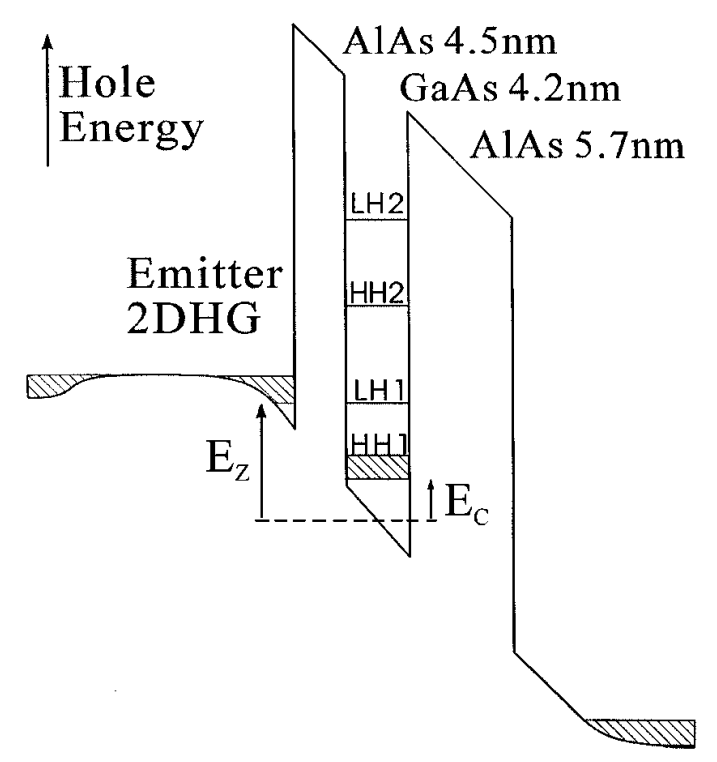

FIG. 1. Schematic band diagram of the device biased close to the LH1 resonance. The 2D subband energy in the emitter is $E_{z}$ and of $\mathrm{HH} 1$ in the QW is $E_{c}$.

(C) 1996 The American Physical Society 


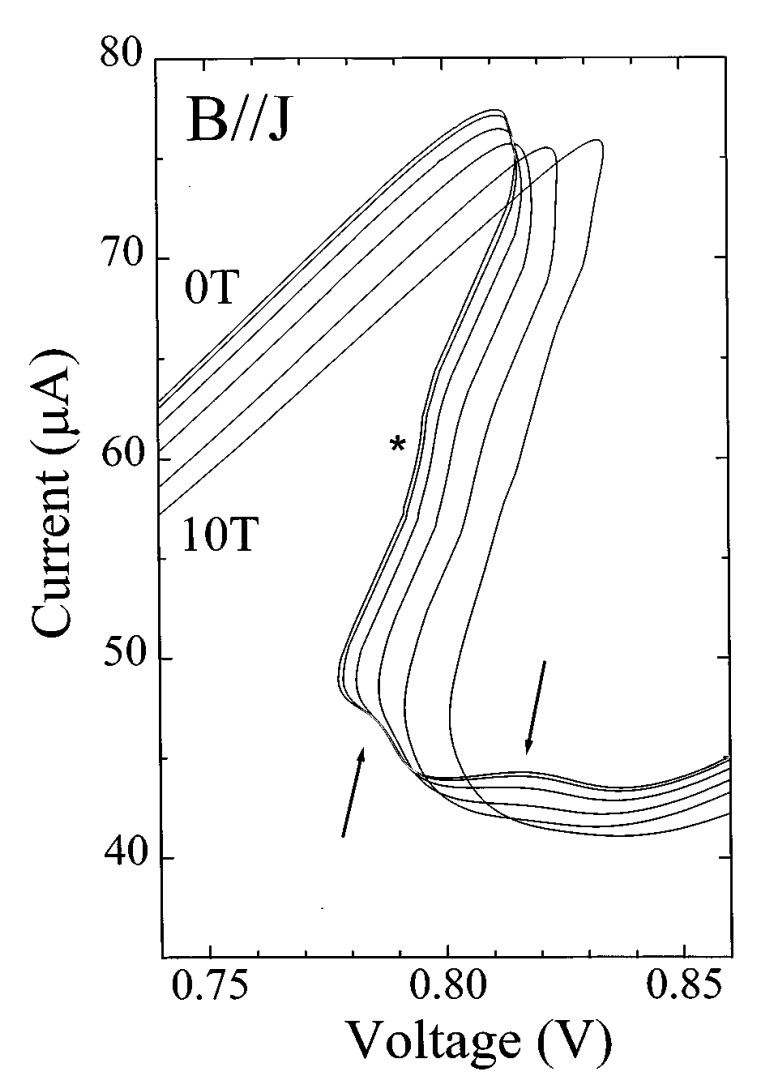

FIG. 2. $I(V)$ characteristics at $4.2 \mathrm{~K}$ in various $\mathbf{B} \| \mathbf{J}$ near the LH1 resonance measured with the NOR circuit. The arrows indicate the plasmon-related features in the $I(V)$ curve at $B=0$. The structure near the asterisk is due to circuit instability. Successive curves are at 2-T intervals.

totunneling measurements on the same device. In the presence of high magnetic fields $B$ applied parallel to the tunnel current, magneto-oscillations periodic in $1 / B$ were observed at various values of bias voltage. These provided us with estimates of the sheet density of holes in the emitteraccumulation layer and in the quantum well. It is found that a significant hole density is present in the quantum well at biases beyond the LH1 resonance, even when the device is off resonance. The holes occupy mainly the HH1 QW subband due to rapid thermalization by phonon emission. ${ }^{9}$

Figure 2 shows the $I(V)$ characteristics of the RTD at 4.2 $\mathrm{K}$ in the bias range of the LH1 resonance. The figure also shows the effect on the LH1 resonance of $\mathbf{B}$ applied along the direction of current flow $(\mathbf{B} \| \mathbf{J})$. This is the only resonance which shows the intrinsic bistability effect. The current at the lower bias HH1 resonant peak (not shown) is very small and exhibits no bistability effect. The full $I(V)$ curve between 0 and $2 \mathrm{~V}$ is shown in Ref. 7. The NOR circuit allows us to probe the voltage overhang region, which is inaccessible with a conventional voltage sweep circuit. Two satellite features can be observed within the overhang region of the main resonance. These are indicated by arrows in Fig. 2. At the bias of the first satellite peak, holes tunnel into the quantum well at energies above that of the LH1 subband, even though the voltage position of the satellite feature is slightly below that of the LH1 resonant peak. This apparent anomaly is caused by the electrostatic effect of hole space-charge buildup in the quantum well on resonance, ${ }^{2,6}$ which is the origin of the Z-shaped resonant peak. In the region of current falloff, a weak shoulderlike feature is present in $I(V)$ at low values of $B$ and $I \approx 60 \mu \mathrm{A}$ (indicated by an asterisk). This is an artefact due to high-frequency oscillations of the current through the circuit, since it is impossible to stabilize the circuit over the entire range of bias, even when the NOR technique is used. ${ }^{6}$ However, the circuit is stable elsewhere, particularly in the regions of interest, i.e., the resonant peak and the satellite features beyond it.

To describe the plasmon-assisted resonant tunneling of holes, we develop the approach used successfully to explain a similar process in electron tunneling. ${ }^{5}$ The model includes explicitly the coupling between the holes tunneling from the emitter accumulation layer and the plasmon excitations of the degenerate hole gas in the HH1 subband of the quantum well. Note that, although the voltage across the device is well beyond the HH1 resonance, hole relaxation in the well still leads to a high population of this subband. The tunneling probability is written as

$$
T\left(E_{k}\right)=\frac{\Gamma_{L} \Gamma_{R}}{\Gamma} \int_{-\infty}^{\infty} \frac{d \sigma}{\hbar} \exp \left[\frac{-\Gamma|\sigma|}{2 \hbar}+i\left(E_{z}-E_{c}\right) \frac{\sigma}{\hbar}-\alpha\right]
$$

with

$$
\begin{aligned}
\alpha= & -\sum_{q} \frac{V_{q}}{\pi} \int_{-\infty}^{\infty} \hbar d \omega\left[1+N_{q}(\omega)\right] \operatorname{Im}\left(\frac{1}{\epsilon(q, \omega)}\right) \\
& \times\left(\frac{i \sigma / \hbar}{\hbar \omega-\Delta}+\frac{e^{-i \sigma(\hbar \omega-\Delta)}-1}{(\hbar \omega-\Delta)^{2}}\right),
\end{aligned}
$$

where $\Gamma_{L}\left(\Gamma_{R}\right)$ is the bandwidth due to tunneling through the left (right) barrier and $\Gamma=\Gamma_{L}+\Gamma_{R}$. The 2D subband energy in the emitter is $E_{z}$, and of HH1 in the QW is $E_{c}$. For the tunneling hole, the energy change in the plane of the quantum well is $\Delta=E_{k}-E_{k-q}$ where $q$ and $k$ are, respectively, the wave vectors of the plasmon and the tunneling hole in the plane of the QW. $N_{q}(\omega)$ is the boson distribution function and $\epsilon(q, \omega)$ is the dielectric function of the system. $V_{q}$ is the Fourier transform of the Coulomb interaction energy between the holes in terms of the plasmon wave vector and $\sigma$ $=t-s$, where $t$ and $s$ are the times for which the interaction occurs for the two particles. The summation over $q$ is limited to $q<q_{F}^{c}$ where the plasma cutoff wave number is $q_{F}^{c}$ (Ref. 5) above which the plasmon is heavily damped.

To apply Eq. (1) to hole tunneling we define the energy $E_{\mathrm{HL}}=E(\mathrm{LH} 1)-E(\mathrm{HH} 1)$ and we assume that there is only a significant population of the HH1 subband. Unlike the electron case ${ }^{5}$ where only one plasmon-assisted process was observed, for holes we have two QW subbands (HH1 and LH1) relatively close in energy. Therefore, when the system is biased such that the energy of holes in the emitter is slightly higher than $E(\mathrm{LH} 1)$, we have to consider two plasmonassisted processes; (i) a hole of energy $E_{H}$ tunnels into the LH1 subband, disposing of its extra energy by the emission of an intrasubband heavy-hole plasmon of frequency $\omega_{\mathrm{HH}}$ so that $E_{H}=E(\mathrm{LH} 1)+\hbar \omega_{\mathrm{HH}}$; (ii) a similar process, this time emitting an intersubband plasmon of frequency $\omega_{\mathrm{HH}}^{*}$ so $E_{H}=E(\mathrm{LH} 1)+\hbar \omega_{\mathrm{HH}}^{*}$ and $\hbar \omega_{\mathrm{HH}}^{*}=\left[\hbar^{2} \omega_{\mathrm{HH}^{2}}+E_{\mathrm{HL}}^{2}\right]^{1 / 2}$. An intersubband plasmon excitation involves the motion of 


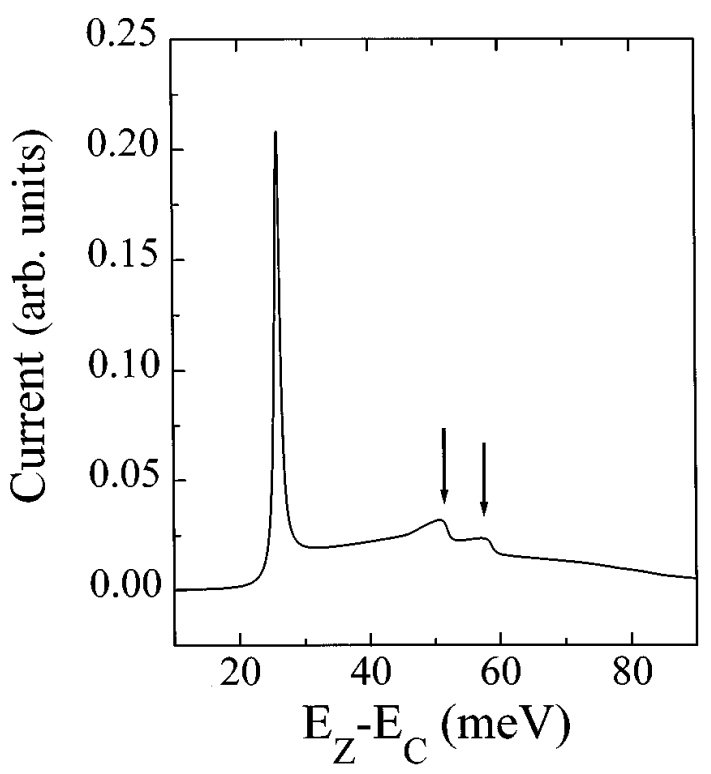

FIG. 3. Calculated tunneling current as a function of the energy difference $E_{z}-E_{c}$ (see Fig. 1). This energy difference can be related to the bias voltage $V$ by the expression $E_{z}-E_{c}=e V / \beta$, where $\beta$ is the electrostatic leverage factor.

charge perpendicular to the plane of the quantum well, although it should be noted that for the HH1-LH1 plasmon, the envelope functions of the two states are very similar. We ignore any contribution from the light-hole plasmon since the population of this subband is measured to be low.

To obtain a qualitative and quantitative picture of the peak separation and the relative strength of processes (i) and (ii), we use a simplified model in which both light holes and heavy holes are treated as free and ideal twodimensional hole gases. Band mixing and finite wave function spreading along $z$ are both neglected. Assuming intrasubband and intersubband modes are well separated in energy, the imaginary part of the inverse dielectric function including both intrasubband and intersubband plasmons can be written as ${ }^{5}$

$$
\begin{aligned}
\operatorname{Im} \frac{1}{\epsilon(q, \omega)}= & \frac{\pi}{2}\left\{\omega_{\mathrm{HH}}\left[\delta\left(\omega-\omega_{\mathrm{HH}}\right)-\delta\left(\omega+\omega_{\mathrm{HH}}\right)\right]\right. \\
& \left.+\frac{\omega_{\mathrm{HH}}^{2}}{\left(\omega_{0}^{2}+\omega_{\mathrm{HH}}^{2}\right)^{1 / 2}}\left[\delta\left(\omega-\omega_{\mathrm{HH}}^{*}\right)-\delta\left(\omega+\omega_{\mathrm{HH}}^{*}\right)\right]\right\}
\end{aligned}
$$

where $\omega_{0}=E_{\mathrm{HL}} / \hbar$. The tunneling current $I$ can be calculated using Eqs. (1) and (2) and integrating over energy. ${ }^{5}$ For the calculation we took $E_{\mathrm{HL}}=20 \mathrm{meV}, \hbar \omega_{\mathrm{HH}}=16\left(q / q_{c}\right)^{1 / 2}$ $\mathrm{meV}, \Gamma=1 \mathrm{meV}$, and $\epsilon_{r}=12$. Hole densities in the emitter and the well were taken to be equal at $2 \times 10^{15} \mathrm{~m}^{-2}$ as measured previously in these devices. ${ }^{9,10}$ The theoretical results for $T=0$ are plotted in Fig. 3. Two resonant satellite peaks are predicted (marked by arrows in Fig. 3) due to intrasubband and intersubband plasmon-assisted tunneling. The lower-energy satellite peak represents tunneling of holes with the excitation of intrasubband plasmons in the HH1 state in the well. The excitation of the more energetic inter-

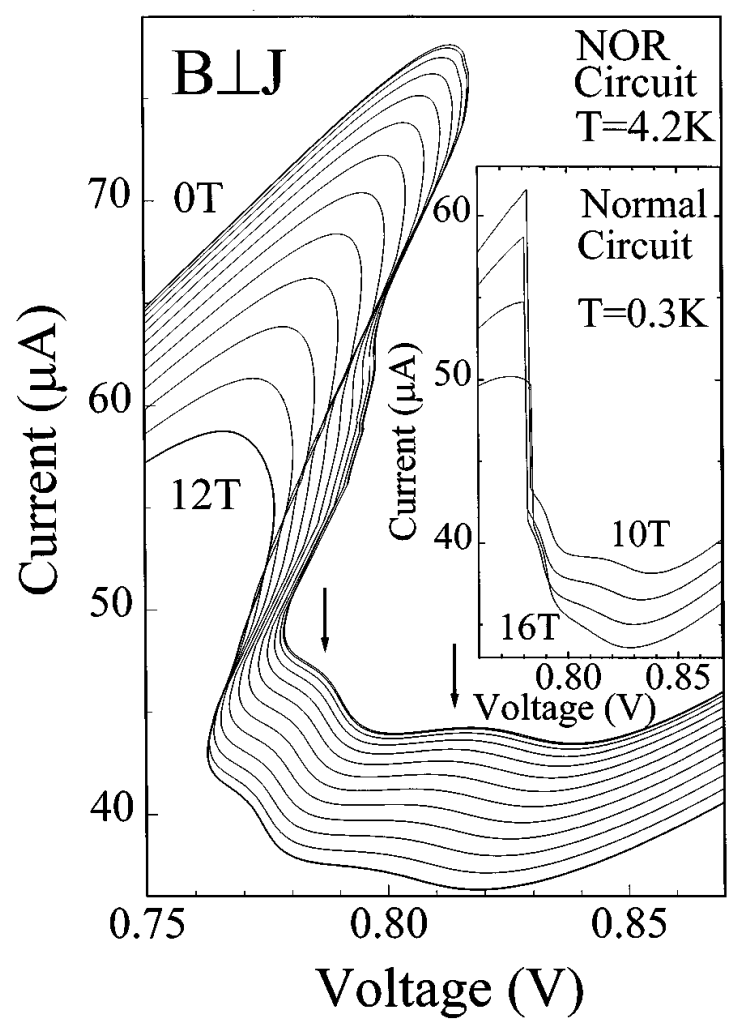

FIG. 4. $I(V)$ characteristics at $4.2 \mathrm{~K}$ measured with a NOR circuit with $\mathbf{B} \perp \mathbf{J}$ for $0<B<12 \mathrm{~T}$ at $1-\mathrm{T}$ intervals. The arrows have the same meaning as in Fig. 2. The inset shows $I(V)$ measured with a conventional circuit for $T=300 \mathrm{mK}$ in $B=10,12,14$, and $16 \mathrm{~T}$.

subband plasmons is responsible for the second satellite peak in Fig. 3. There is reasonable quantitative agreement between experiment and theory for the relative sizes and voltage positions of the two satellites of the LH1 resonance. Using the experimentally determined value ${ }^{9}$ of $\beta$, the ratio of the voltage drop across the device to energy difference between the emitter and the well, we estimate the satellite peaks at $B=0$ of Fig. 2 to be separated by $\sim 32 \mathrm{mV}$, corresponding to an energy difference of $5 \mathrm{meV}$. This compares reasonably well with the theoretical spacing of $\sim 7 \mathrm{meV}$ in Fig. 3. It is difficult to compare the satellite spacing with the bias position of the LH1 resonance due to the difference between the hole charge density in the quantum well on the LH1 resonance and on the satellites. However, the qualitative similarity between Figs. 2 and 3 supports the interpretation that both satellite features in the $I(V)$ curve arise from plasmon-assisted tunneling of holes.

In order to investigate the satellites further, we have studied the effect of magnetic field $\mathbf{B}$, oriented either parallel to or perpendicular to the current. The effect of $\mathbf{B} \| \mathbf{J}$ is shown in Fig. 2. There is a small shift of the main resonance towards higher bias and both plasmon peaks are apparently quenched for $B>7 \mathrm{~T}$. At this magnetic field, the plasmon mode is strongly modified by cyclotron motion. Note that the magnetic-field behavior for the plasmon satellites is quite different from that of the satellite associated with tunneling accompanied by the emission of a longitudinal-optic (LO) phonon. ${ }^{4}$ In that case the quantization into Landau lev- 
els of the carrier motion in the quantum well leads to an increase in the strength of the LO phonon satellite as $B$ increases. $^{11}$

The effect on the satellites of $\mathbf{B}$ perpendicular to the current $(\mathbf{B} \perp \mathbf{J})$ is much weaker and is shown in Fig. 4. The main effect of $\mathbf{B}$ in this configuration is to decrease the amplitude of the main LH1 resonance, which is also shifted towards lower bias. This bias shift can be explained by conservation of canonical momentum in the tunneling process and by the LH1 energy-wave-vector dispersion curve, which shows electronlike (negative hole effective mass) behavior. ${ }^{11}$ This causes the resonant peak to shift to lower bias with increasing $B$. The reduction of the amplitude of the LH1 resonance may be related to the increasing barrier heights at the tunneling energy which accompany the shift of the resonance condition to lower bias. However, the voltage positions of the plasmon satellites should not shift: first, the magnetic field in the plane of the QW perturbs the plasmon excitation only slightly since the field does not couple to the in-plane collective motion; secondly, canonical momentum of the tunneling electron is not conserved for plasmon-assisted tunneling. In addition, the magnetic length $\ell_{B}$ is large compared to the
QW width so that the magnetic field will not affect significantly the coupling of the $\mathrm{HH}$ and LH subbands. This probably accounts for the persistence of the two plasmon satellites up to $12 \mathrm{~T}$ for $\mathbf{B} \perp \mathbf{J}$. Measurements made at $0.3 \mathrm{~K}$ with a conventional bias circuit (inset of Fig. 4) show the presence of both satellites for $\mathbf{B} \perp \mathbf{J}$ up to $16 \mathrm{~T}$, although there is some indication that the peaks are weaker at this field. (For $\mathbf{B} \perp \mathbf{J}>12 \mathrm{~T}$, it is possible to observe both features with a conventional circuit due to the shift and decrease in amplitude of the main LH1 resonant peak).

In summary, we have observed satellite peaks due to holeplasmon-assisted resonant tunneling processes in the currentvoltage characteristics of a $p$-type double barrier heterostructure. The magnetic field dependence of these satellites is investigated, described and discussed qualitatively. A simple theoretical model is presented which accounts quantitatively for the strength in comparison to the principal hole resonant peak.

This work was supported by EPSRC. B.R.A.N. and L.E. acknowledge CNPq (Brazil) and EPSRC for financial support, respectively.
${ }^{1}$ C. J. Powell and J. B. Swan, Phys. Rev. 115, 869 (1959).

${ }^{2}$ M. L. F. Lerch, A. D. Martin, P. E. Simmonds, L. Eaves, and M. L. Leadbeater, Solid-State Electron. 37, 961 (1994).

${ }^{3}$ E. S. Alves, L. Eaves, M. Henini, O. H. Hughes, M. L. Leadbeater, F. W. Sheard, G. A. Toombs, G. Hill, and M. A. Pate, Electron. Lett. 24, 1190 (1988).

${ }^{4}$ V. J. Goldman, D. C. Tsui, and J. E. Cunningham, Phys. Rev. Lett. 58, 1256 (1987).

${ }^{5}$ C. Zhang, M. L. F. Lerch, A. D. Martin, P. E. Simmonds, and L. Eaves, Phys. Rev. Lett. 72, 3397 (1994).

${ }^{6}$ A. D. Martin, M. L. F. Lerch, P. E. Simmonds, and L. Eaves, Appl. Phys. Lett. 64, 1248 (1994).
${ }^{7}$ D. A. Broido and L. J. Sham, Phys. Rev. B 31, 888 (1985).

${ }^{8}$ G. Bastard, Wave Mechanics Applied to Semiconductor Heterostructures (Les Éditions de Physique, Paris, 1988).

${ }^{9}$ R. K. Hayden, L. Eaves, M. Henini, D. K. Maude, and J. C. Portal, Phys. Rev. B 49, 10745 (1994).

${ }^{10}$ R. K. Hayden, L. Eaves, M. Henini, D. K. Maude, J. C. Portal, and G. Hill, Appl. Phys. Lett. 60, 1474 (1992).

${ }^{11}$ R. K. Hayden, D. K. Maude, L. Eaves, E. C. Valadares, M. Henini, F. W. Sheard, O. H. Hughes, J. C. Portal, and L. Cury, Phys. Rev. Lett. 66, 1749 (1991); R. K. Hayden, L. Eaves, M. Henini, T. Takamasu, N. Miura, and U. Ekenberg, Appl. Phys. Lett. 61, 84 (1992). 